\title{
A no-reference image quality measure based on CPBD and noise
}

\author{
Yufeng Gu ${ }^{1,}$, Kejian Yang ${ }^{1, b}$ \\ ${ }^{1}$ Computer Science and Technology, Wuhan University of Technology, Wuhan, Hubei 430063, \\ China \\ a854987418@qq.com, bykjcgs@whut.edu.cn
}

Keywords: No-reference; Image Quality Assessment; CPBD; Noise pollution.

\begin{abstract}
In order to meet the needs of the objective evaluation of no-reference image, a no-reference image quality measure is presented. The measure is based on edge analysis and is suitable for images with noise. Taking properties of the Human Visual System(HVS) into account, we compute the probability of blur after getting the edge width and the local contrasts. And at last the image quality probability can be got considering cumulative probability of blur detection and the noise pollution degree. Experimental results show that the metric has a wide application, good anti-noise ability, simple calculation, as well as in high consistence with the subjective evaluation results.
\end{abstract}

\section{Introduction}

A With the widespread use of image information technology, the image which contains a lot of valuable information is more and more valued as the source of visual information. In the process of image acquisition and transmission, it is inevitable that images will be affected by noise, blur, the loss of data and so on, resulting in a decrease of image quality. The image quality directly affects the amount of information acquired and the subjective feelings of people. So, an automated and objective no-reference image quality evaluation assessment is needed considering the errors caused by subjective judgment and the time-consuming staff.

So far research on reference and semi-reference image quality evaluation has achieved good results. And the non-reference evaluation which is most practical has gradually become the researches emphasis in recent years. Some scholars have studied the image quality evaluation method, and have achieved some results.

These algorithms are either based on edge analysis to evaluate the image blurriness as in literature [1-4], or simply estimate the variance of the noise as in [7-10]. For images which contain blur and noise in the same time, these motheds lack the ability to evaluate them. Considering the situation, this paper proposes an evaluation method combining the edge analysis and image noise level, which can be used more broadly.

\section{The Improved Quality Probability Model}

To evaluate the quality of an image with blur and noise, an improved probability model based on the edge analysis is proposed by taking noise into account. In this section, the implementation procedures of the algorithm are given in detail. 


\subsection{Edge Detection and Edge Width Measurement.}

The better the image is, the more obvious the edges are, and the narrower the edge width is. So the edge width largely reflects the sharpness of an image. In general, the difference is used to find the gradient, and the gradient direction is obtained. Then the local extremum can be find by searching along the two ends of the gradient direction. And the distance between the two local extremum is the edge width. But to reduce the computational complexity and ensure reasonably accurate measurements, only the approximately vertical edges are added to the calculation, for the literature [11] points out that incorporating extra horizontal measurements do not improve the performance. And considering the measurements of diagonal edge gradients will conversely result in a decreased performance which is due to the larger quantization width $(\sqrt{2})$ of diagonal pixels compared with horizontal/vertical direction. The edge width $w\left(e_{i}\right)$ is computed as follows:

$$
w\left(e_{i}\right)=\frac{I_{\max \left(e_{i}\right)^{-I} \min \left(e_{i}\right)}}{\cos \left(\Delta \emptyset\left(e_{i}\right)\right)} .
$$

Where $I_{\max \left(e_{i}\right)}$ and $I_{\min \left(e_{i}\right)}$ are local maximum and local minimum of the edge pixel $e_{i}$, see Fig. 1. $\Delta \emptyset\left(e_{i}\right)$ denotes the angle difference between the gradient $\emptyset\left(e_{i}\right)=\tan ^{-1}\left(I_{y\left(e_{i}\right)} / I_{x}\left(e_{i}\right)\right)$ direction and the vertical direction, where $I_{x}\left(e_{i}\right)$ and $I_{y\left(e_{i}\right)}$ are the image gradient components. Although the denominator in Eq. 1 compensates for angle difference, large deviations will contribute to errors in the measurement process. Thus, only approximately' vertical edges which satisfy the conditions of $\Delta \emptyset\left(e_{i}\right)<\Delta \emptyset_{\max }$ are considered. Experiments show that low thresholds $\Delta \emptyset_{\max } \leq 2^{\circ}$ will decrease performance as there are no enough measurements to be representative, and larger thresholds $\Delta \emptyset_{\text {max }} \geq 15^{\circ}$ also decreases performance, due to the error propagation affected by imprecise measurements. The thresholds $\Delta \emptyset_{\max }=8^{\circ}$ can lead to a good performance.

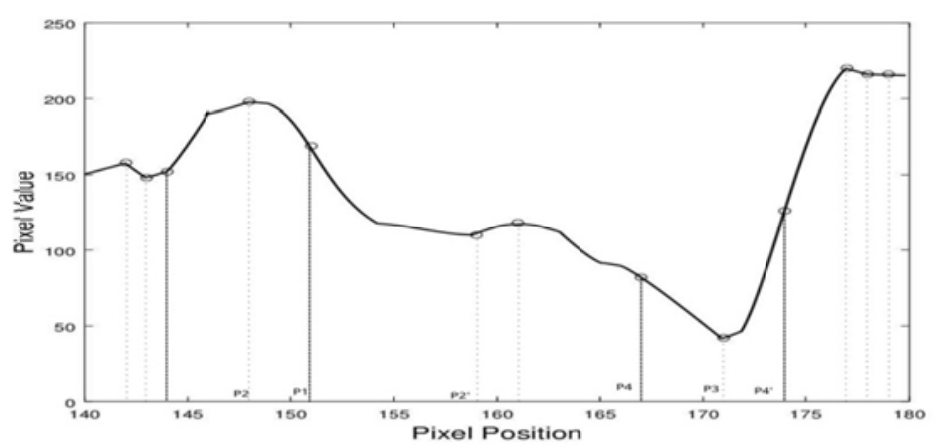

Fig. 1. Part of an image line. The wide dotted lines indicate the detected edges. The narrow dotted line represents the local maxima $I_{\max \left(e_{i}\right)}$ and local minimum $I_{\min \left(e_{i}\right)}$.

\subsection{Cumulative Probability Model of Blur Detection.}

The Just Noticeable Difference (JNB) concept is proposed in [5] based on the Human Visual System (HVS). It defines the minimum amount of perceived blurriness around an edge by giving a contrast. For a given contrast c, a probability of blurriness can be calculated as follows:

$$
p_{\text {blur }}=p\left(e_{i}\right)=1-\exp \left(-\left|\frac{w\left(e_{i}\right)}{w_{J N B}\left(e_{i}\right)}\right|^{\beta^{\prime}}\right) \text {. }
$$

Where $\mathrm{w}_{\mathrm{JNB}}\left(\mathrm{e}_{\mathrm{i}}\right)$ is the just noticeable blurred edge width depended on the local contrast. The parameter $\beta$ is obtained by using the least squares curve fitting method for different contrasts $c$ in 
order to increase the consistency between Eq. 2 and the experimental results of the fuzzy psychological perception. The experimental results in literature [6] find that the probability is more accurate and credible when $\beta$ has a median value of 3.6, and the JNB edge widths for different contrasts can be modeled as:

$$
\mathrm{w}_{\mathrm{JNB}}\left(\mathrm{e}_{\mathrm{i}}\right)=\left\{\begin{array}{ll}
5, & \mathrm{c} \leq 50 \\
3, & \mathrm{c} \geq 51
\end{array} .\right.
$$

An improved method of JNB is proposed in literature [9]. The method divides an image into $64 \times 64$ blocks. Then the blocks whose edge pixels are accounted for more than $0.2 \%$ of the total number of pixels in the block are defined as edge blocks to be processed further. It is pointed out that, at the JNB with, $w\left(e_{i}\right)=w_{J N B}\left(e_{i}\right)$ corresponds to a probability of blur detection $p_{\text {blur }}=$ $\mathrm{P}_{\mathrm{JNB}}=63 \%$. Thus, for a given edge pixel $\mathrm{e}_{\mathrm{i}}$, when $\mathrm{p}_{\mathrm{blur}} \leq \mathrm{P}_{\mathrm{JNB}}$, it is considered that there is no blur to be detected at that edge. With the increase of the blur degree of an image, the edges' width increases, which leads to a bigger value of $w\left(e_{i}\right)$ and to a higher probability of blur detection. Considering all of the edge blocks and the edges, the entire image's sharpness probability is modeled as a cumulative probability of blur detection (CPBD) as follows:

$$
\mathrm{CPBD}=\mathrm{P}\left(\mathrm{p}_{\text {blur }} \leq \mathrm{P}_{\mathrm{JNB}}\right)=\sum_{\mathrm{p}_{\text {blur }}=0}^{\mathrm{p}_{\text {JNB }}} \mathrm{P}\left(\mathrm{p}_{\text {blur }}\right) \text {. }
$$

Where $P\left(p_{\text {blur }}\right)$ is defined as the value of the probability distribution function at a given $p_{\text {blur }}$.

\subsection{Measurement of Noise Effects.}

Noise, as one of the most common image degradation factors, is often produced in the process of generation and propagation of an image. When evaluating the image quality with noise, people pay more attention to the intensity of noise rather than it's random characteristic, for it is the intensity of noise that effects people's subjective perception. The differences between the images before and after denoising reflect the noise pollution degree. Thus, after using the total variation (TV)[10] minimization method which can save the image details as much as possible to denoise, the signal noise ratio(SNR) which denotes the ratio of the original image signal intensity and the intensity of noise can be got :

$$
\mathrm{SNR}=10 \cdot \log _{10} \frac{\sum_{\mathrm{i}=1}^{\mathrm{M}} \sum_{\mathrm{j}=1}^{\mathrm{N}} \mathrm{f}(\mathrm{i}, \mathrm{j})^{2}}{\sum_{\mathrm{i}=1}^{\mathrm{M}} \sum_{\mathrm{j}=1}^{\mathrm{N}}[\mathrm{g}(\mathrm{i}, \mathrm{j})-\mathrm{f}(\mathrm{i}, \mathrm{j})]^{2}} .
$$

Where $M$ and $N$ are the size of the image. $f(i, j)$ denotes the denoised image and $g(i, j)$ denotes the original image with noise. Considering the randomness of the noise, the probability that the detected edges are effected by the noise to reduce the quality of the image is $1 /(S N R+1)$, that is, the ratio of the image quality to be reduced.

\subsection{The Comprehensive Evaluation Probability Model.}

There is a CPBD model which denotes the probability of edge sharpness of the whole image and the ratio noise affects the quality of an image. Since the noise is random and independent of the image signal or the edge sharpness, a new probability model can be obtained by putting them together:

$$
\mathrm{P}=\frac{\mathrm{SNR}}{\mathrm{SNR}+1} \cdot \mathrm{CPBD} \text {. }
$$

Where $\mathrm{P}$ is the final quality evaluation value, that is, the probability of a high quality image. And the higher the image quality is, the higher the value will be. 


\section{Experimental Results}

In this section, experimental results are provided to illustrate the performance of the proposed no-reference image quality measure. The proposed metric was tested with images with different contents obtained from the LIVE database[12].
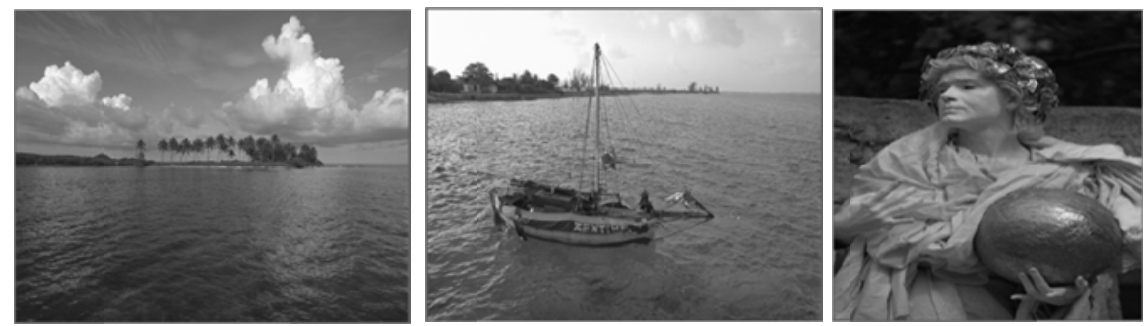

Fig. 2. Test images

As the blurriness increase, the probability of blur detection is expected to increase, and the image quality is reduced, leading to a lower probability score. Fig. 3(a) shows the performance of the proposed method for three groups images(Fig. 2) which are being Gauss blurred in LIVE. Since there is no single image that is being Gauss blurred with continuous interleaved standard deviations, we blurred images with a circular-symmetric 2D Gaussian kernel of standard deviation $\sigma_{b l u r}$, which ranges from 0 to 8 with the step size 0.2. Fig. 3(b) shows the result of evaluating the generated images with the proposed metric for the series of caps images. The same two experiments were carried out on all the tested images, and a similar performance was observed. From Fig. 3 (a) and (b), it can be found that the proposed metric is behaving as expected. With the standard deviation increase, the probability decrease rapidly. When the standard deviation is about 3.0, the score is almost 0 , for almost all the edge widths are greater than the JNB width and the image become bad.

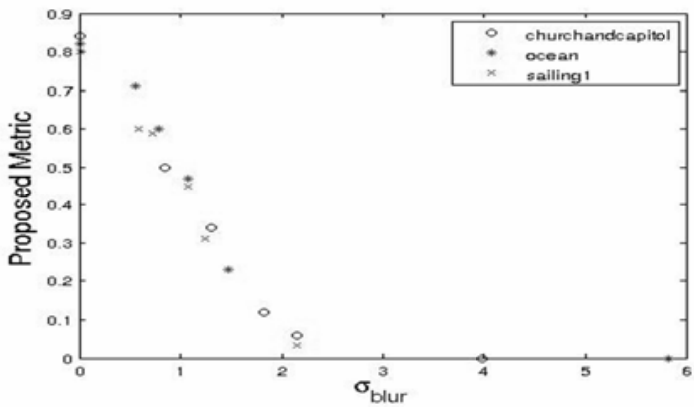

(a)

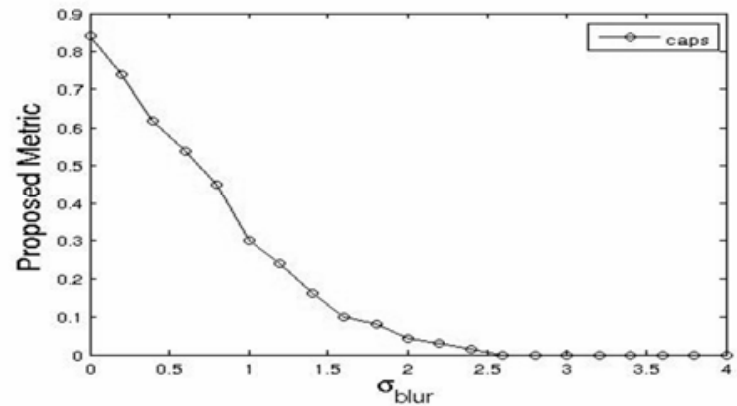

(b)

Fig. 3. The performance of the proposed method tested on (a) blurred images in LIVE and (b) caps image blurred with different $\sigma_{\text {blur }}$.

To evaluate images with noise, noise effect is added into the assessment model. The performance of the proposed method about noise was tested against other three metrics: DMOS, PSNR, CPBD. The result is shown in Fig. 4. The Difference Mean Opinion Score (DMOS) denotes the difference of the subjective value between the original image and the distorted image. And the larger the DMOS is, the worse the image quality is. 
Table 1. standard deviation sigma of white noise and corresponding score

\begin{tabular}{|l|l|l|l|l|l|l|}
\hline sigma & 0.0000 & 0.019531 & 0.035156 & 0.140625 & 0.234375 & 1.1996094 \\
\hline DMOS & 0.000 & 24.376 & 33.502 & 47.815 & 53.846 & 57.678 \\
\hline PSNR & $+\infty$ & 38.9170 & 33.9107 & 22.2460 & 18.3455 & 10.0190 \\
\hline CPBD & 0.8446 & 0.8135 & 0.8362 & 0.8641 & 0.9053 & 0.9249 \\
\hline Our & 0.8224 & 0.7568 & 0.6472 & 0.3964 & 0.2316 & 0.0487 \\
\hline
\end{tabular}

The Table 1 shows that the CPBD method is only based on the edge width to give a probability of blur, which is lack of the ability to evaluate the noise level in the image. Due to the impact of noise on the edge detection and calculation of the edge width, when the image is polluted heavily by noise, the CPBD method even completely lost the reference. But the proposed method takes into account the noise effect resulting that when the image is polluted heavily by noise, the ratio of noise effect increases and dominates and corrects the final score making it possible that the result obtained is consistent with the subjective evaluation of HVS to accurately evaluate the quality of the image with noise.

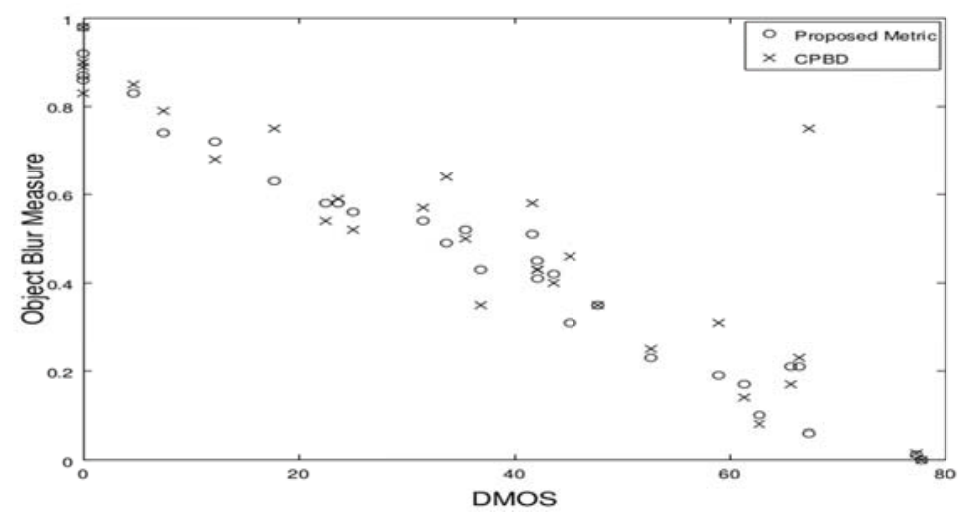

Fig. 4. The performance of CPBD and proposed metric on different types of image distortion.

At last, we test the performance of the proposed method on other types of image distortion. With six images picked in each type of distortion, 30 images are selected from all the five distortion types to form a set of images whose DMOS distribution is relatively wide and uniform. Using the CPBD and proposed metric to evaluate these images, the result shows in Fig. 4. Through the experiment, it can be found that the proposed method has a more accurate evaluation ability for all kinds of distorted images in LIVE. Comparing with CPBD, it is closer to the subjective perception and has a lower volatility.

\section{Conclusions}

Image quality affects the acquisition of information, thus, a general and accurate method for the objective evaluation of no reference image is deeply needed. In this paper, the proposed method improves the CPBD model by taking into account the influence of noise. The test results show that the proposed algorithm is close to the subjective perception of the quality of all kinds of images, and it is possessed of stronger applicability and generality.

\section{References}

[1] Ong E P, Lin W, Lu Z, et al. A no-reference quality metric for measuring image blur[C]// Signal Processing and Its Applications, 2003. Proceedings. Seventh International Symposium on. IEEE, 2003:469-472 vol.1.

[2] Ong E P, Lin W, Lu Z, et al. No-reference JPEG-2000 image quality metric[C]// Multimedia and Expo, 2003. 
ICME '03. Proceedings. 2003 International Conference on. IEEE, 2003:I-545-8 vol.1.

[3] Yan Q, Xu Y, Yang X. No-reference image blur assessment based on gradient profile sharpness[C]// Broadband Multimedia Systems and Broadcasting (BMSB), 2013 IEEE International Symposium on. IEEE, 2013:1-4.

[4] Wang $\mathrm{Y}, \mathrm{Du} \mathrm{H}, \mathrm{Xu}$ J, et al. A no-reference perceptual blur metric based on complex edge analysis[C]// Network Infrastructure and Digital Content (IC-NIDC), 2012 3rd IEEE International Conference on. IEEE, 2012:487-491.

[5] Ferzli R, Karam L J. Human Visual System Based No-Reference Objective Image Sharpness Metric[C]// IEEE International Conference on Image Processing. 2006:2949-2952.

[6] Ferzli R, Karam L J. A no-reference objective image sharpness metric Based on just-noticeable blur and probability summation[C]// IEEE International Conference on Image Processing. 2007(3): III-445-III-448

[7] Immerkær J. Fast Noise Variance Estimation[J]. Computer Vision \& Image Understanding, 1996, 64(2):300-302.

[8] Laligant $\mathrm{O}$, Truchetet F, Fauvet E. Noise estimation from digital step-model signal.[J]. IEEE Transactions on Image Processing A Publication of the IEEE Signal Processing Society, 2013, 22(12):5158-67.

[9] Narvekar N D, Karam L J. A no-reference perceptual image sharpness metric based on a cumulative probability of blur detection[C]// Quality of Multimedia Experience, 2009. QoMEx 2009. International Workshop on. 2009:87 - 91.

[10] Rudin L I, Osher S, Fatemi E. Nonlinear total variation based noise removal algorithms[J]. Physica D Nonlinear Phenomena, 1992, 60(1-4):259-268.

[11] Feichtenhofer C, Fassold H, Schallauer P. A Perceptual Image Sharpness Metric Based on Local Edge Gradient Analysis[J]. IEEE Signal Processing Letters, 2013, 20(4):379-382.

[12] H. R. Sheikh, Z. Wang, L. Cormack and A. C. Bovik, "LIVE Image Quality Assessment Database Release 2", http://live.ece.utexas.edu/research/quality. 